\title{
Estudio retrospectivo comparativo entre los estados IIIB clínico y el homologado a IIIB por Tx de las pacientes con cáncer de seno en el INC
}

\author{
Antonio Luis Visbal M. MD*; Celso Rodríguez Sánchez MD**; Maritza Rey Pinzón***
}

RESUMEN: En el INC hay un grupo importante de pacientes que al llegar a la consulta el tumor ha sido manipulado; basados en experiencia histórica los pacientes manipulados presentan menor período libre de enfermedad y sobrevida, se decidió clasificar a estos pacientes como Tx y homologarlos IIIB. Existe la preocupación que estos pacientes se encuentren en un estado clínico diferente. Este estudio diseñado con el fin de encontrar diferencias en relación con sobrevida, período libre de enfermedad y metástasis entre los pacientes en un estado clínico IIIB y los homologados, encontró que los pacientes manipulados eran más jóvenes; no encontró diferencia en cuanto al tamaño del tumor al momento del ingreso, características clínicas de T4, compromiso axilar, características histopatológicas, metástasis, período libre de enfermedad y sobrevida. Sinembargo, no fue posible reclasificar el $40 \%$ de los pacientes al momento del ingreso según el TNM por falta de las características clínicas necesarias, el $\mathbf{4 0} \%$ presentaban características clínicas de T4 y fueron reclasificados como IIIB (80\% de este subgrupo fue imposible distinguir si el edema de piel se debía a manipulación quirúrgica previa o a infiltración tumoral); al compararlos con los IIIB clínico y los demás Tx-IIIB tuvieron igual sobrevida y PLE. Un $20 \%$ de los homologados tenían características TNM al momento del ingreso de un estado más temprano; encontrándose diferencia en sobrevida y PLE como subgrupo.

A la manipulación el médico sólo tuvo en cuenta la clasificación TNM en 10/76 pacientes y sólo 3 correspondían al mismo estado al ingreso. No se encontró diferencia en sobrevida al comparar los IIIB con los Tx-IIIB teniendo en cuenta el tiempo transcurrido entre la manipulación y el momento del ingreso, esto no indica que la manipulación afecte la sobrevida porque desconocemos el estado clínico al momento de la manipulación en el $89 \%$ de los casos y el número de los pacientes restantes no soportan un análisis estadístico confiable.

PALABRAS CLAVES: TX, III-B, Tx-IIIB, manipulación.

SUMMARY: In the NC there is an important group of patients upon arriving to the conference the tumor has been manipulated; based on historical experience the manipulated patients present smaller period of free of disease and survival; there fore it was decided to classify to these patients as Tx and to homologate them as IIIB. Exist the preoccupation that these patients are found in a different clinical state. This retrospective descriptive study designed in order to find differences in relationship to survival, period free of disease and metastasis between the patients in a clinical state IIIB and the homologateed, found that the manipulated patients were but young; it did not find difference with respect to size of the tumor moment of the revenue, characteristic clinics of T4, commitment axilar, hitostopatologics characteristic, metastasis, period free of disease and survival. It was not possible to reclassify $40 \%$ of the patients to the moment of the revenue according to the TNM by lack of the characteristic necessary clinics, $40 \%$ were presenting characteristic clinics of T 4 and they were reclassifyed as IIIB $(80 \%$ of this subgroup was impossible to distinguish if the skin edema had to be to previous surgical manipulation or to tumoral infiltration); upon comparing them with the clinical IIIB and the other Tx-IIIB equal survival and period free of disease was obtained. A $\mathbf{2 0 \%}$ of the homologateed characteristic had TNM to the moment of the revenue of an earliest state; it being found difference in survival and period free of disease as subgrupo.

To the manipulation the alone physician took into account the classification TNM in 10/76 patients and only 3 were corresponding to the same state to the revenue. It was not found difference in survival upon comparing the IIIB with the Tx-IIIB taking into account the time elapsed between the manipulation and the moment of the revenue, this not indicate that the manipulation affect the survival because do not know the clinical state to the moment of the manipulation in $89 \%$ of the cases and the number of the remaining patients do not sustain a reliable statistic analysis.

KEY WORDS: TX, III-B, TX-IIIB, manipulation.

\section{Introducción}

Actualmente se considera al $\mathrm{Ca}$ de seno como una enfermedad sistémica desde sus inicios, basados en el cambio del enfoque Halstediano del cáncer por Fisher (3), el hallazgo de ganglios axilares positivos en un $30 \%$

R. IV Cirugía General.

** Cirujano Oncólogo, Servicio Cirugía General y Seno.

*** Grupo de Estudios Epidemiológicos INC. de pacientes con tumores no palpables (diagnosticados por mamografía) y la recurrencia a distancia hasta 20 años o más después de un tratamiento curativo (2). E1 número de ganglios axilares es el factor predictivo más importante de sobrevida a diez años en $\mathrm{Ca}$ invasor. Fisher demostró (4) que la sobrevida neta y el intervalo libre de enfermedad están afectados por el número de ganglios axilares comprometidos. Se ha visto que si no existen ganglios axilares la sobrevida a cinco años es del $78 \%$ y a diez del $65 \%$. Por el contrario si hay 4 ó más, la sobrevida a cinco años cae al $32 \%$ y a los diez años al 
13\%. También se ha encontrado relación entre el número de ganglios linfáticos comprometidos y la falla al tratamiento a los cinco años, encontrándose en un $20 \%$ a diez años cuando no hay ganglios positivos y $71 \%$ cuando hay 4 ó más ganglios positivos. Los factores histopatológicos más importantes son: la presencia de ganglios axilares positivos, la clasificación histológica, el grado del tumor, la presencia de receptores esteroideos positivos, la tasa de crecimiento, la cinética tumoral; y la presencia de marcadores biológicos.

Características histológicas del tumor, se han establecido cuatro grupos (5):

A. Todas las lesiones In Situ, con una sobrevida a cinco años del $91 \%$.

B. Ca Medular, Ca Mucinoso, Ca Tubular y $\mathrm{Ca}$ Adenoide quístico, con sobrevida del $75 \%$, a cinco años.

C. Ca Ductal Infiltrante y Ca Lobular Infiltrante, con sobrevida del $65 \%$.

D. Ca Inflamatorio y $\mathrm{Ca}$ Indiferenciado con una sobrevida del $33 \%$ a cinco años.

En cuanto al grado Histológico, se ha estudiado el tamaño, la forma y el hipercromatismo nuclear, así como el porcentaje y número de mitosis y túbulos. Se han desarrollado grados histológicos de acuerdo a las características nucleares solamente y su diferenciación, encontrándose una sobrevida a 20 años así: Grado 1 (bien diferenciado) $49 \%$, Grado 2 (moderadamente diferenciado) $29 \%$ y Grado 3 (pobremente diferenciado) $21 \%$.

Fisher encontró una relación significativa entre el grado histológico del tumor y la sobrevida a cinco años (6).

Para el análisis histopatológico del tumor se utilizan los criterios del TNM clínicos aplicados a la pieza quirúrgica anteponiéndose la sigla $p$. Se requiere que no haya evidencia macroscópica de tumor en las márgenes de sección y que por lo menos esté completo el primer nivel de disección axilar. Cuando el tumor se encuentra presente en los márgenes de sección del espécimen se le aplica al código $\mathrm{Tx}$, indicándose la imposibilidad para determinar el estado $\mathrm{T}$ patológico. Tx es una clasificación patológica que requiere de la extracción de la pieza quirúrgica para su análisis. Si los ganglios axilares han sido extraídos previamente y no se pueden clasificar se le asigna la nomenclatura $\mathrm{Nx}$.

El propósito de la estadificación, para un $\mathrm{CA}$, es poder determinar la extensión anatómica de la enfermedad, la tendencia a la progresión, y brindar una terapia adecuada dentro de unos protocolos que han demostrado mejores resultados y puedan ser comparables con los aplicados entre diferentes instituciones.

El cáncer de seno es la primera causa de mortalidad en mujeres en países desarrollados, su incidencia se encuentra en incremento y se considera que al $12 \%$ de la población femenina se le diagnosticará Ca de seno en el transcurso de su vida (1). En Colombia se estima una Incidencia de 27.1 por 100.000 mujeres, siendo la tercera causa de Mortalidad por cáncer en la población general y la segunda en la femenina (12-13). En el INC, es la segunda causa de consulta por cáncer, y el mayor porcentaje se encuentran en estados IIIB (39.2\%) (12-13). Un número importante de pacientes remitidos al INC para tratamiento, han sido previamente manipulados con fines diagnósticos y/o tera- péuticos (18.5\%), en la mayoría de los casos, no han sido clasificados en su correspondiente estado clínico, antes de iniciar algún tipo de intervención. Tradicionalmente se han considerado pacientes tratados en forma inadecuada por violación de las barreras biológicas; además en los controles históricos de los pacientes manipulados se encontraron casos que presentaron metástasis más tempranas en comparación con el estado correspondiente al momento del ingreso. En consideración a lo anterior se decidió tratar a los pacientes manipulados igual al estado localmente más avanzado, por lo que se Homologan a IIIB. El tratamiento de los pacientes con Ca de Seno IIIB es quimioterapia no adyuvante, de acuerdo con la respuesta clínica sigue a cirugía o radioterapia preoperatoria y luego cirugía (que en la mayoría de los casos es una Mastectomía Radical Modificada, la Mastectomía Simple Ampliada se reserva para los pacientes mayores con buena respuesta a la radioterapia preoperatoria). De acuerdo con el estudio patológico del seno se continúa con quimioterapia adyuvante o terapia hormonal (11). Los pacientes homologados a IIIB se tratan igual.

Recientemente en la consulta de seno del INC se han visto casos de pacientes homologados con una mejor sobrevida que la correspondiente a un estado IIIB, considerándose la posibilidad que hayan sido sobretratados con deterioro de su calidad de vida. Con el presente estudio se desea comparar los pacientes con cáncer de scno estado IIIB clínico y los pacientes homologados a este estado en cuanto a su evolución clínica, respuesta al tratamiento y sobrevida.

\section{Justificación}

En el Servicio de Cirugía General y Seno del Instituto Nacional de Cancerología de Colombia se reciben anualmente un número importante de pacientes que han recibido algún tipo de manipulación previa con fines diagnósticos o terapéuticos antes de ser remitidos.

Históricamente se ha visto que alguno de estos pacientes presentaban recaída más temprana y menor sobrevida si se los comparaba con los pacientes correspondientes al estado clínico en el momento de la manipulación y al momento del ingreso; de otra parte se han encontrado casos anecdóticos de pacientes manipulados con una sobrevida mejor que la correspondiente al estado IIIB. Esto hizo cuestionar si a este grupo de pacientes al momento de la manipulación fueron clasificados en el estado clínico correcto, si los hallazgos al examen físico del seno al momento del ingreso son confiables o si la violación de la técnica quirúrgica influye negativamente en estos pacientes; adicionalmente un porcentaje importante de estos pacientes no habían sido clasificados según el TNM en algún estado clínico antes de la manipulación sobre el tumor. Con estas dudas se decidió que estos pacientes fueran clasificados como $\boldsymbol{T} \boldsymbol{x}$ y se homologaran al estado clínico localmente más avanzado, IIIB. Sinembargo, no se conoce si la evolución clínica a largo plazo de estos pacientes es realmente igual, mejor o peor a la del estado al cual se han homologado; y no existe un estudio que apoye la conclusión que los pacientes homologados a un estado IIIB tienen una evolución 
clínica mejor en comparación con pacientes del mismo estado clínico.

\section{Objetivos}

1. Comparar la evolución clínica de los pacientes clasificados como Tx, homologados a IIIB, con los pacientes IIIB clínicos tratados en el INC en términos de metástasis a distancia, período libre de enfermedad y sobrevida.

2. Identificar si los pacientes homologados a IIIB por Tx alteran la tasa de metástasis, período libre de enfermedad y sobrevida del total de pacientes IIIB.

3. Identificar si comparando factores histológicos como el tamaño macroscópico del tumor, presencia de ganglios axilares, grupo y grado de diferenciación histológica hay diferencia en relación con sobrevida y período libre de enfermedad entre el grupo de pacientes IIIB y los homologados a IIIB.

4. Identificar si el tipo de manipulación y el tiempo transcurrido desde la remisión hasta que el paciente es visto por primera vez en la consulta de seno del INC son factores negativos relacionados con sobrevida y período libre de enfermedad en el grupo de pacientes TxIIIB.

\section{Hipótesis}

En el Instituto Nacional de Cancerología de Colombia los pacientes con Cáncer de Seno clasificados Tx homologados a IIIB tienen una evolución clínica distinta en relación con período libre de enfermedad; incidencia de metástasis y sobrevida, si se compara con las pacientes en el estado clínico IIIB.

\section{Diseño metodológico}

Se realizó un estudio Retrospectivo Descriptivo. Se incluyeron en el estudio todos los pacientes con cáncer de seno de sexo femenino que fueron atendidos por primera vez entre enero $1^{\circ}$ de 1989 y diciembre 31 de 1990 en la consulta de seno del INC y se encontraban en el estados clínicos IIIB; así como aquellos que son remitidos al INC entre estas fechas y habían recibido algún tipo de manipulación previa sobre el tumor y/o la axila, y fueron homologados al estado IIIB (TxIIIB o NxIIIB). No entraron en el estudio los pacientes que se les hubiese practicado cualquier tipo de Mastectomía o Cuadrantectomía (con o sin Vaciamiento Axilar) y los que se les hubiese practicado Vaciamiento Axilar como parte del tratamiento para cáncer de seno y fueron remitidos al INC para tratamiento complementario; así como los pacientes que recibieron IIIB (Tx o clínico) que no iniciaron el tratamiento indicado.

La fuente de datos fue la historia clínica donde se extrajeron y se consignaron en un formulario diseñado para tal fin.

Se revisaron las historias clínicas de los pacientes estadificados como IIIB y de ellos se tomaron para el estudio los que cumplieron con los criterios de inclusión, analizándose la hoja de ingreso al INC, la hoja de remisión, la hoja de ingreso al servicio de seno, la hoja quirúrgica, el resultado de histopatología, el tratamien- to adyuvante (Qtx y Rtx), buscándose los datos a estudiar, consignándolos en un formulario diseñado para este estudio.

Se revisaron los treinta y un datos a estudiar anotando hallazgos en la hoja de recolección posteriormente se tabularon en una base de datos y luego se practicaron cruces entre estos con el fin de encontrar la relación entre las variables analizadas. Se estudiaron tanto los pacientes en estado IIIB clínico como los IIIB homologados en relación con sobrevida, recurrencia locorregional, metástasis y período libre de enfermedad. Se compararon los resultados analizando si hubo una mejor sobrevida de los pacientes homologados en relación con los IIIB clínico y como se afectó la sobrevida cuando se suman los TxIIIB con los IIIB clínicos.

Se clasificaron los pacientes homologados con el posible estado clínico al momento de la manipulación y al momento del ingreso acorde con el TNM, y se trató de comparar con la sobrevida de series publicadas correspondientes a este estado.

El tratamiento estadístico de la información se hizo con los programas EPIINFO 6.03 y SAS versión 6.0 para Windows. La significancia estadística se fijó en alfa menor a 0.05. Para comparar los promedios se utilizó el Test de $t$ y para la comparación de proporciones el Test de Chi Cuadrado. Para la estimación de sobrevida y tiempo libre de enfermedad se hizo por el método de Kapplan-Meier y para la comparación de ellas se utilizó el Test de Log Rank.

\section{Variables}

Edad: Del paciente en años.

Tx-. Tumores de pacientes remitidos al INC, que se consideran manipulados previamente.

Estado IIIB: Pacientes que al ingreso al servicio de seno del INC presentan las características clínicas para ser estadificados en este grupo.

TxIIIB: Pacientes que al ingreso al servicio de seno del INC han sido previamente manipulados sobre el seno y son homologados al estado III.

Manipulación: Biopsia incisional, biopsia excisional, biopsia por trucut. Cuando el paciente se le practique una tumorectomía se asimilará como biopsia excisional.

Tiempo de remisión: Período desde la fecha de manipulación y la fecha de ingreso al servicio de seno.

Tamaño del tumor a la manipulación: Medida del diámetro mayor en $\mathrm{cm}$. al momento de la manipulación.

Características Clínicas de T4 a la manipulación: Compromiso de piel, pared torácica o $\mathrm{Ca}$ Inflamatorio al momento de la manipulación.

Compromiso Axilar a la manipulación: Presencia de ganglios comprometidos por tumor según el TNM clínico al momento de la manipulación.

Tamaño del tumor al ingreso: Medida del diámetro mayor en $\mathrm{cm}$. al ingreso

Características Clínicas de T4 al ingreso: Compromiso de piel, pared torácica o $\mathrm{Ca}$ inflamatorio.

Compromiso Axilar al ingreso:Presencia de ganglios comprometidos por tumor según el TNM clínico al momento del ingreso. 
Tamaño del tumor en la pieza quirúrgica: Medida del diámetro mayor del tumor en $\mathrm{cm}$ de la pieza microscópica.

Presencia de Tumor en Pieza quirúrgica: Positividad histológica para cáncer en el producto de la mastectomía radical modificada o simple ampliada luego de haber recibido tratamiento neoadyuvante (Quimioterapia, $\mathrm{Ra}$ dioterapia o ambas).

Compromiso Axilar en la pieza quirúrgica: Presencia de ganglios comprometidos en número o de la grasa axilar en el estudio anatomopatológico de la pieza quirúrgica.

Tipo Histológico: De acuerdo con las características histológicas del tumor divididos en cuatro grupos: Todas las lesiones In Situ Grupo A; Ca Medular, Mucinoso, Tubular y Adenoide Quístico, Grupo B; Ca Ductal Infiltrante y Lobulillar Infiltrante, Grupo C; y Ca Inflamatorio e Indiferenciado Grupo D (5).

Período libre de enfermedad: Tiempo durante el cual no hay evidencia clínica de recidiva local o a distancia.

Sobrevida: Tiempo de vida total del paciente.

Recurrencia Local: Presencia de tumor en el seno luego de haber sido terminado el tratamiento y declarado clínicamente libre.

Recurrencia Regional: Presencia de tumor en la axila luego de haber terminado el tratamiento y considerado clínicamente libre.

Metástasis: Evidencia clínica de tumor en fosas supraclaviculares, seno contralateral o en otros organos, luego de haberse terminado el tratamiento.

\section{Resultados}

Se revisaron $392 \mathrm{HC}$ de los pacientes Estadificados como IIIB que fueron atendidos por primera vez en la consulta de seno en el período del estudio, para tener un seguimiento mínimo de 66 meses y máximo de 84 meses. Se eliminaron 184 pacientes por no tener los criterios de inclusión: 44 por habérseles practicado MRM en otra institución, 40 por no haber iniciado el tratamiento indicado en el INC, 30 por estar mal clasificados y tener como primario un tumor distinto al Ca de seno, 20 por no encontrarse entre las fechas del estudio, 10 por habérseles practicado radioterapia en otra institución, 9 por haber recibido quimioterapia en otra institución, 2 por habérseles practicado Cuadrantectomía y VR en otra institución, 1 por haber sido sometido a MSA en otro hospital y 2 por presentar patología benigna de seno.

Para el estudio quedaron 208 pacientes con 209 tumores (un paciente de los homologados recibió manipulación en ambos senos). De este número de pacientes 132 (63.2\%) se encontraron en el estado IIIB clínico y 76 fueron homologados a IIIB por Tx.

El rango de edad varió entre 10 y 85 años para la población de estudio, con un promedio de 51.3 años y una mediana de 50 años. Los pacientes IIIB tenían una edad promedio de 52.5 años al momento del ingreso al estudio y una mediana de 50 años. Los pacientes homologados tenían una edad promedio de 49.1 años y una mediana de 46 años; encontrándose una diferencia significativamente estadística para la edad $(\mathrm{p}=0,049)$.
CARCINOMA DE SENO IIIB Y IIIB HOMOLOGADO. DISTRIBUCION POR GRUPOS ETAREOS

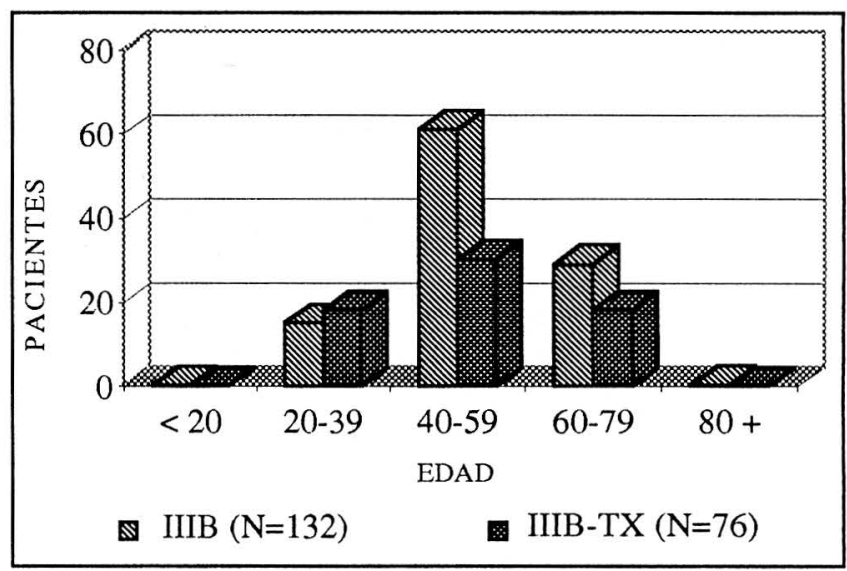

El tipo de manipulación realizado más frecuente fue la biopsia excisional en 49 oportunidades $(63.6 \%)$, seguidos por la biopsia incisión en 19 (24.7\%), biopsia con aguja tru-cut 2, biopsia con tru-cut y excisional 1, biopsia con tru-cut e incisión 2 y en 3 pacientes no se encontró qué tipo de manipulación se practicó.

La cicatriz fue la secuela dejada más frecuente por la manipulación $97.4 \%$ de las veces ( 75 casos) y en el $2.6 \%$ de las veces ( 2 casos) cicatriz con esquimosis.

En cuanto al tamaño del tumor para el grupo de estudio se encontraron 4 pacientes con tamaño menor o igual a dos cms. (1.9\%), 85 entre 2.1 y $5 \mathrm{cms}$. (40.7\%), 70 entre 5.1 y $8 \mathrm{cms}$. (33.5\%), 12 entre 8.1 y $11 \mathrm{cms}$. (5.7\%) y 3 entre 11.1 y $13 \mathrm{cms}$. (1.5\%). En 35 pacientes (16.7\%) no se encontró presencia de masa al examen físico de ingreso. El tamaño promedio al ingreso de los IIIB fue de $5.7 \mathrm{cms}$. con una mediana de $5.5 \mathrm{cms}$; para los pacientes homologados fue de $5.4 \mathrm{cms}$. con una mediana de 5.0 cms; no encontrándose diferencia estadísticamente significativa $(\mathrm{p}=0.544)$.

De los 76 pacientes manipulados (77 senos con tumor), no se encontró el tamaño al momento de la manipulación en 38 (49.4\%). De los 39 casos restantes (50.6\%), en 6 se reporta menor o igual a $2 \mathrm{cms}(15.4 \%), 20$ entre 2.1 y $5 \mathrm{cms}$. (51.3\%), 10 entre 5.1 y $8 \mathrm{cms}$. (25.6\%) y en 3 entre 8.1 y $10 \mathrm{cms}$. (7.7\%).

El tamaño promedio del tumor al ingreso para los Tx fue de $5.4 \mathrm{cms}$. con una variance de 4.90 y a la manipulación fue de $4.8 \mathrm{cms}$. con una variance de 4.73. Al comparar el tamaño del tumor al ingreso con el tamaño del tumor a la manipulación en los Tx IIIB se encontró una diferencia estadísticamente significativa para aumento del tamaño desde el momento de la manipulación hasta el ingreso al INC ( $t 1.69$, con de $\mathrm{p}=0.197$ - valor $p=0.544$ ), sinembargo hay que anotar que sólo se pudo tomar los datos de la mitad de los pacientes manipulados.

En los 209 senos estudiados (208 pacientes) 38 casos (18.2\%) no presentaban signos de T4 al momento del ingreso, $165(79 \%)$ compromiso de piel (dado por edema, infiltración y/o ulceración), 4 por compromiso de piel y fijación a pared torácica (1.9\%), 1 por fijación a pared torácica y 1 por $\mathrm{Ca}$ inflamatorio. 
Analizando los signos de $\mathrm{T} 4$ al momento del ingreso en cada uno de los grupos se encontró que en los pacientes IIIB $126(95.6 \%)$ eran T4b, $4(3 \%)$ T4c, 1 T $4 a(0.7 \%)$ y $1 \mathrm{~T} 4 \mathrm{~d}(0.7 \%)$; en los pacientes homologados 38 casos no presentaron signos de $\mathrm{T} 4 \mathrm{al}$ ingreso $(50 \%), 30$ presentaron edema de piel que no se podía diferenciar entre infiltración tumoral o cambios postquirúrgicos (40\%) y en 9 se encontraron signos de T4b indudable (10\%).

$\mathrm{Al}$ momento de la manipulación en 59 casos no se reportan datos acerca del estado de la piel o fijación a pared torácica, en 13 casos no se reportan signos de T4, en 2 se reportan signos de $\mathrm{T} 4 \mathrm{a}$ y en 3 signos de $\mathrm{T} 4 \mathrm{~b}$.

En relación con el estado clínico de la axila al ingreso como grupo se encontró que en 55 casos (26.3\%) la axila se clasificó como N0, en $72(34.4 \%)$ como N1 y en 81 (39.3\%) como N2. Al analizarlos por grupos se encontró N0 en 28 pacientes IIIB (21.3\%), N1 en 41 (31\%) y N2 en $63(47.7 \%)$; mientras que para los Tx-IIIB se encontró N0 en $27(35 \%)$, N1 en $31(40.15 \%)$ y N2 en $19(24.75 \%)$.

Cuando se analizan los datos de los Tx-IIIB al momento de la manipulación se encuentra que en 57 casos (74\%) no se sabe el estado de la axila; de los restantes se clasifican clínicamente como N0 en 13 casos (16\%), N1 en $6(7.8 \%)$ y $\mathrm{N} 2$ en $1(1.3 \%)$.

No se pudo reclasificar los tumores de los pacientes homologados según el TNM del ingreso por falta de los datos necesarios en 31 casos $(40.3 \%)$; 4 se reclasificaron a IIA (5.2\%), 5 a IIB (6.5\%), 6 a IIIA (7.8\%) y $31(40.3 \%)$ permanecieron como IIIB.

Al aplicar el TNM a los datos de la hoja de remisión en donde se describen las características clínicas del tumor al momento de la manipulación no se pudo identificar el estado clínico en 64 casos ( $83 . \%$ ), de los 13 casos restantes 5 quedaron como IIIB, 2 como IIIA, 1 como IIB, 3 como IIA, 2 como Estado Uno; sinembargo si se comparan los datos de estos pacientes con la reclasificación clínica al momento del ingreso sólo 2 de los IIIB quedarían como tal, uno no se podría clasificar por ausencia de masa y no presentar signos de IIIB al ingreso y los otros 2 pasarían a IIIA, de los demás cambian excepto por 1 paciente en Estado Uno. El número de estos pacientes es muy pequeño para cualquier tipo de análisis, sinembargo si consideramos los seis pacientes con estados hasta máximo IIB la mitad están muertos.

TABLA DE RECLASIFICACION CLINICA

\begin{tabular}{|lll|}
\hline HC & E. a la manipulación & E. al ingreso \\
258570 & IIIA & IIIB \\
259389 & IIB & IIIB \\
259941 & IIIB & IIIA \\
262489 & IIA & IIIA \\
263567 & IIIB & IIIB \\
263567 & UNO & UNO \\
263389 & IIIB & IIIA \\
260941 & IIA & IIIB \\
253984 & UNO & NO SE PUEDE \\
$\mathbf{2 5 3 0 8 1}$ & IIIB & IIIB \\
257481 & IIA & IIIB \\
253065 & IIIB & NO SE PUEDE \\
251768 & IIIA & IIIB \\
\hline
\end{tabular}

TABLA DE RECLASIFICACION CLINICA AL MOMENTO DE LA MANIPULACION DE LOS CINCO PACIENTES EN ESTADOS “TEMPRANOS"

\begin{tabular}{|llllr|}
\hline HC & $\begin{array}{l}\text { Estado } \\
\text { Clínico }\end{array}$ & $\begin{array}{l}\text { Sobrevida } \\
\text { (meses) }\end{array}$ & $\begin{array}{l}\text { Estado último } \\
\text { Control }\end{array}$ & Vivo \\
259389 & IIB & 53.86 & Enferma & No \\
263567 & I/IIIB & 55.23 & Enferma & No \\
$\mathbf{2 6 0 9 4 1}$ & IIA & $\mathbf{6 3 . 2 6}$ & Libre & $\mathbf{S i}$ \\
$\mathbf{2 5 3 9 8 4}$ & UNO & $\mathbf{8 3 . 2 6}$ & Libre & $\mathbf{S i}$ \\
$\mathbf{2 5 7 4 8 1}$ & IIA & $\mathbf{5 6 . 0 0}$ & Libre & $\mathbf{S i}$ \\
262489 & IIA & 50.50 & Enferma & No \\
\hline
\end{tabular}

\section{CARCINOMA DE SENO IIIB HOMOLOGADO. RECLASIFICACION CLINICA}

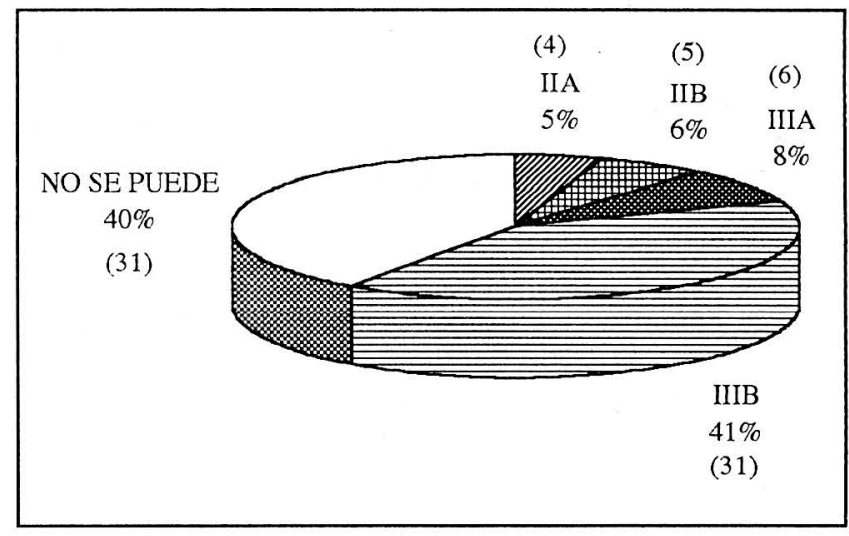

De los 176 pacientes que fueron a cirugía (111 IIIB y $65 \mathrm{Tx}$-IIIB) luego de tratamiento neoadjuvante (quimioterapia más radioterapia o viceversa o sólo radioterapia o quimioterapia) el tamaño promedio del tumor en la pieza de patología fue de $4.917 \mathrm{cms}$. con una mediana de 4 y un rango de 1 a 17 . El tamaño del tumor promedio para los IIIB fue de $4.8 \mathrm{cms}$. con una mediana de 4 y para los Tx-IIIB de $5.0 \mathrm{cms}$. con una mediana de $4.0 \mathrm{cms}$. No encontrándose diferencia estadísticamente significativa $(\mathrm{p}=0.79)$.

En relación con la presencia de tumor en la pieza de patología se encontró que en los IIIB no hubo presencia de tumor en $30.6 \%$ de los casos contra un $46.1 \%$ de los Tx-IIIB. Se encontró presencia de tumor en un cuadrante en el $51.2 \%$ de los IIIB y en el $35.9 \%$ de los Tx-IIIB. Se encontró presencia de tumor en más de dos cuadrantes en un porcentaje pequeño para ambos grupos. Aunque hubo un mayor porcentaje de negativización de la pieza patológica en los pacientes homologados no hubo una diferencia significativamente estadística $(\mathrm{p}=0.125)$.

En relación con el análisis de los bordes se encontró bordes negativos para el $83 \%$ de los estados IIIB (92/111) y $89.5 \%$ para los Tx-IIIB (58/65). El borde de sección profunda fue positivo para el $3.6 \%$ de los IIIB (4/111) y el 3\% de los Tx-IIIB (2/65). La presencia de borde de piel sobre el tumor positivo fue del $8.1 \%$ para los IIIB (9/111) 
y del 6\% para los Tx-IIIB (4/65). La presencia de borde de sección profundo y piel sobre el tumor fue positiva en $4.4 \%$ de los IIIB (5/111) y del $1.5 \%$ de los Tx-IIIB (1/65). El borde de resección fue positivo en piel en un $0.9 \%$ (1/ 111) de los pacientes IIIB. No se encontró diferencia estadísticamente significativa en cuanto a la presencia o ausencia de bordes en los dos grupos.

En relación con el tipo histológico en el $89.5 \%$ de los casos fueron tumores correspondientes al grupo C (168 Ductales, 19 Lobulillares), el 6.2\% al grupo B (1 Adenoide Quístico, 1 Mucinoso, 5 Coloides, y 5 Tubulares), el $2.9 \%$ fueron del grupo D (6 Ca Inflamatorios), $1 \%$ del grupo A ( 2 Lobulillares In Situ) y un tumor Phyllodes (manipulado).

En los Ductales infiltrantes el $72 \%$ fueron bien diferenciados, el $14 \%$ moderadamente diferenciados y el $12 \%$ mal diferenciados.

En los 26 pacientes IIIB que presentaban axila negativa al ingreso y fueron a cirugía 20 se encontraron negativas, 6 se encontraron positivas ( 2 con 1 a 3 ganglios y 4 con 4 o más ganglios o infiltración a la grasa axilar). En los 18 pacientes Tx-IIIB con axila negativa que fueron a cirugía 12 se encontraron igual, 5 con 1 a 3 ganglios positivos y 1 con 4 o más ganglios positivos o infiltración a la grasa axilar). De los 86 pacientes IIIB que presentaron axila clínicamente positiva y fueron a cirugía 28 se encontraron negativas, en 19 se encontraron de 1 a 3 ganglios positivos y en 29 se encontraron 4 o más ganglios o infiltración a la grasa axilar. En los pacientes Tx-IIIB con axila positiva al ingreso que fueron a cirugía (47) se encontraron negativas en 26 , en 10 se encontraron de 1 a 3 ganglios positivos y en 9 se encontraron 4 o más ganglios o infiltración a la grasa axilar.

Los 132 pacientes IIIB terminaron el siguiente tratamiento: 17 sólo recibieron radioterapia preoperatoria y cirugía para continuar con tamoxifén; 75 pacientes recibieron radioterapia preoperatoria y quimioadyuvante seguido de cirugía y quimioterapia adyuvante; 4 pacientes recibieron radioterapia preoperatoria y quimioneoadyuvante seguido de cirugía y quimioterapia adyuvante pero no completaron el tratamiento por toxicidad; 14 pacientes recibieron radioterapia preoperatoria y quimioneoadyuvante seguido de cirugía y quimioterapia adyuvante presentando metástasis durante ésta por lo que se cambió el esquema; 15 pacientes recibieron radioterapia preoperatoria y quimioneoadyuvante seguido de cirugía y quimioterapia adyuvante abandonando el tratamiento durante la quimioadyuvante; 2 pacientes presentaron recurrencia locorregional durante la quimioterapia adyuvante luego de haber recibido radioterapia preoperatoria y quimioneoadyuvante seguido de cirugía; en 6 pacientes sólo se dio quimioterapia neoadyuvante y luego cirugía y en sólo 2 radioterapia y cirugía. De los 76 pacientes homologados $13(17.1 \%)$ recibieron radioterapia preoperatoria y cirugía seguido de tamoxifén; en 41 (53.9\%) recibieron radioterapia preoperatoria y quimioneoadyuvante seguido de cirugía y quimioterapia adyuvante; 2 pacientes $(2.6 \%)$ abandonaron por toxicidad durante la quimioterapia adyuvante (luego de haber recibido radioterapia preoperatoria y quimioneoadyuvante seguido de cirugía); 1 (1.3\%) presentó progresión local de la enfermedad durante la quimioadyuvante, 3 pacientes $(3.9 \%)$ no terminaron por presentar metástasis durante la quimioterapia adyuvante y hubo que cambiar el tratamiento; 15 pacientes (19.9\%) abandonaron el tratamiento durante la quimioterapia adyuvante y 6 recibieron quimioterapia seguida de cirugía. No se encontró diferencia significativamente estadística en relación con el tipo de tratamiento administrado y terminado en ambos grupos, así como el porcentaje de abandono, de progresión local y de metástasis durante el tratamiento.

\section{CARCINOMA DE SENO IIIB. TIPO HISTOLOGICO}

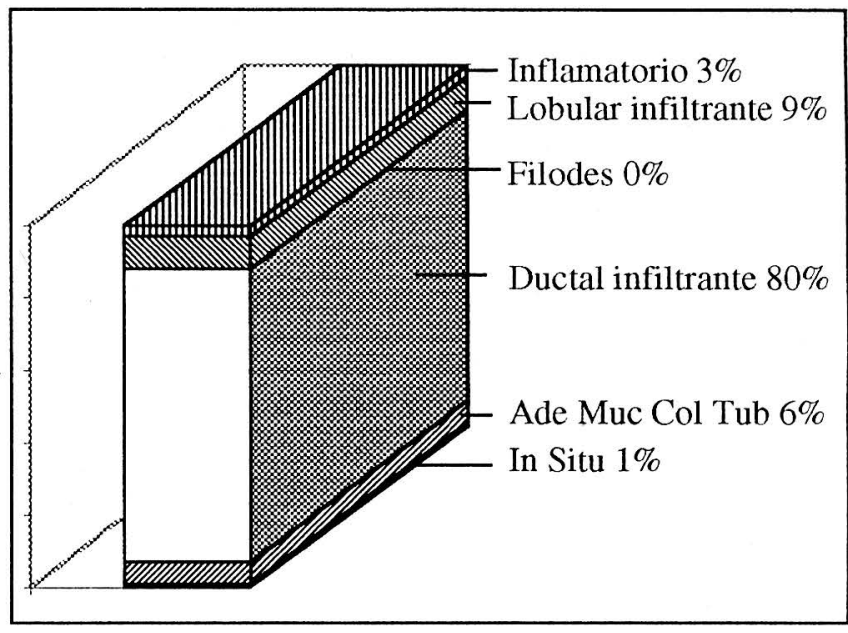

En cuanto al resultado del tratamiento de los 132 pacientes IIIB que terminaron el tratamiento $93(70.4 \%)$ se declararon libres de enfermedad, 9 presentaron persistencia locorregional de la enfermedad al final del tratamiento $(6.8 \%), 15$ evidencia clínica de enfermedad sistémica $(11.3 \%), 14$ tanto persistencia locorregional como evidencia clínica de enfermedad sistémica y 1 abandonó el tratamiento. De los pacientes homologados $54(71.5 \%)$ se declararon libres de enfermedad, $6(7.5 \%)$ continuaban con persistencia locorregional, $8(10.5 \%)$ con evidencia clínica de enfermedad sistémica y $8(10.5 \%)$ con persistencia locorregional y evidencia clínica de enfermedad sistémica.

El porcentaje de pacientes que no presentaron metástasis fue similar para ambos grupos, 87/132 de los IIIB (66\%) y 51/76 de los Tx-IIIB (67\%). El sitio más frecuente de metástasis fue el sistema osea para ambos grupos, $14 / 132$ de los IIIB (10.5\%) y 10/76 de los TX-IIIB $(13.5 \%)$. El segundo sitio en frecuencia de metástasis fue el pulmón $13 / 132$ para los IIIB (10\%), seguido de la fosa supraclavicular ipsilateral 5/132 (3.78\%). El segundo sitio en frecuencia de metástasis para los pacientes homologados fue el cerebro $5 / 77(6.5 \%)$ y luego el pulmón $4 / 77$ (5.19\%).

Cuando se realizó el último control se encontraron clínicamente libres de tumor $75 / 132$ pacientes IIIB $(56.8 \%)$ y $44 / 76$ de los Tx-IIIB $(57.8 \%)$. Habían recaído $57 / 132$ de los IIIB $(43.2 \%)$ y $32 / 76$ de los Tx-IIIB (42.2\%). No se encontró diferencia estadística al comparar los dos grupos $(\mathrm{p}=0.921)$. 
El seguimiento fue similar para los dos grupos, se pudo hacer seguimiento completo en 96 pacientes de 132 en los IIIB (72.7\%) y en 53 de los 77 pacientes TX-IIIB $(69.73 \%) ; p=0.658$. El análisis de sobrevida no mostró diferencia estadística cuando se compararon los dos grupos, encontrándose $85.7 \%$ de los pacientes IIIB vivos a los 24 meses y $83.4 \%$ de los homologados. A los 60 meses el $67.6 \%$ de los IIIB se encontraron vivos y el $71.7 \%$ de los Tx-IIIB (log Rank test $\mathrm{p}=0.267$, ver gráfica de sobrevida). Cuando se compararon ambos grupos teniendo en cuenta la presencia de tumor en la pieza de patología, el compromiso axilar y el grado histológico no se encontró diferencia estadística (como se puede observar en la tabla de sobrevida según hallazgos patológicos).

A los 81.4 meses el $73 \%$ de los pacientes IIIB y el $74 \%$ de los Tx-IIIB permanecen libres de enfermedad como se puede observar en la gráfica de período libre de enfermedad ( $\log$ Rank test, $\mathrm{p}=0.714)$. Al comparar el tiempo libre de enfermedad global con los hallazgos patológicos de presencia de tumor, axila y grado de diferenciación no hubo diferencia estadísticamente significativa (como se puede observar en la tabla de tiempo libre de enfermedad según los hallazgos patológicos).

Cuando se comparan el grupo de pacientes TxIIIB que al momento del ingreso no se pudieron clasificar en ningún estado clínico por la ausencia de masa con los IIIB no hubo diferencia estadística en cuanto a sobrevida $(p=0.266)$ o período libre de enfermedad $(p=0.512)$.

Cuando se comparan el grupo de pacientes Tx-IIIB que al momento del ingreso no se pudieron clasificar en ningún estado clínico por la ausencia de masa con los TxIIIB que se reclasificaron como IIIB no hubo diferencia estadística en cuanto a sobrevida $(p=0.185)$ o período libre de enfermedad $(\mathrm{p}=0.151)$.

Cuando se comparan el grupo de pacientes Tx-IIIB que al momento del ingreso presentaban como característica clínica de T4 edema de piel con los IIIB no hubo diferencia en cuanto a sobrevida $(\mathrm{p}=0.570)$ o período libre de enfermedad $(p=0.330)$.

Cuando se comparan el grupo de pacientes Tx-IIIB que al momento del ingreso se pudieron clasificar estados distintos más tempranos al IIIB con los IIIB si hubo diferencia en cuanto a sobrevida $(\mathrm{p}=0.037)$ no en período libre de enfermedad $(p=0.205)$. Al comparar el mismo grupo de pacientes con los Tx-IIIB reclasificados como IIIB también se encontró diferencia en cuanto a sobrevida (p 0.026) y tuvieron un período libre de enfermedad más prolongado $90.9 \%$ contra $57.7 \%$ sin recaída a los 60 meses $(p=0.065)$.

$\mathrm{Al}$ analizar la variable manipulación previa y compararla con sobrevida se encontró lo siguiente: no se encontró diferencia estadística cuando se comparan entre los Tx-IIIB los manipulados antes de un mes y mayor de un mes previo al ingreso al INC (Test de Log Rank $\mathrm{p}=$ 0.973 ). Al comparar por separado cada uno de los grupos Tx-IIIB con los IIIB clínico tampoco se encontró diferencia estadística (menos de un mes $\mathrm{p}=0.441$, más de un mes $\mathrm{p}=0.419$ Test de Log Rank). Si se comparan los tres grupos de pacientes: estado clínico IIIB con los manipulados antes de un mes previo a su remisión y después de un mes no se encontró diferencia estadística en cuanto a sobrevida (Test de Log Rank $\mathrm{p}=0.590$ ).

\section{CARCINOMA DE SENO IIIB. ESTADO AL FIN DEL TRATAMIENTO}

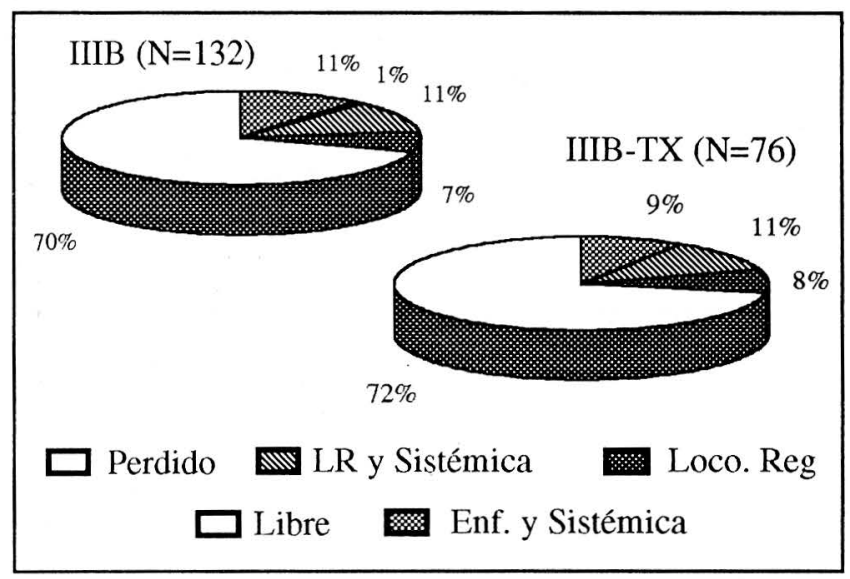

CARCINOMA DE SENO IIIB. METASTASIS

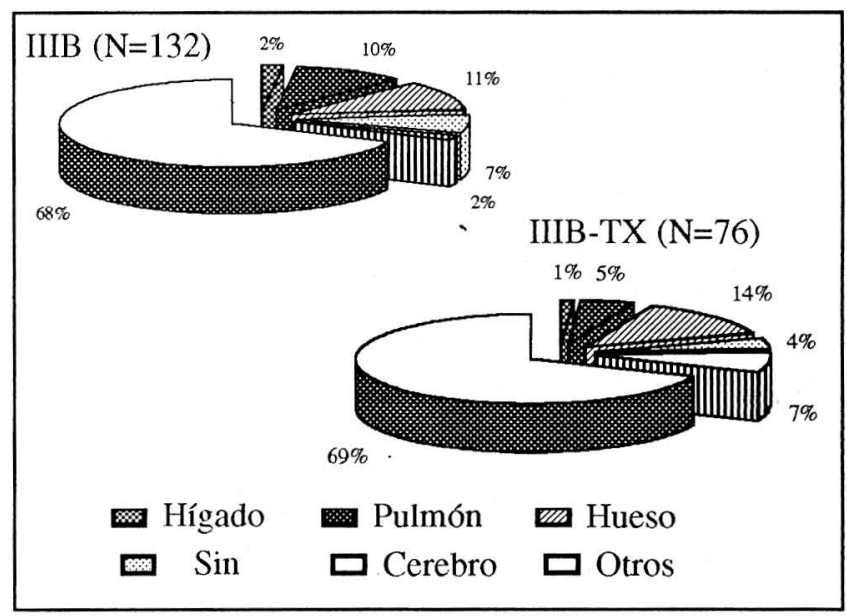

CARCINOMA DE SENO IIIB. SOBREVIDA SEGUN CLASIFICACION CLINICA

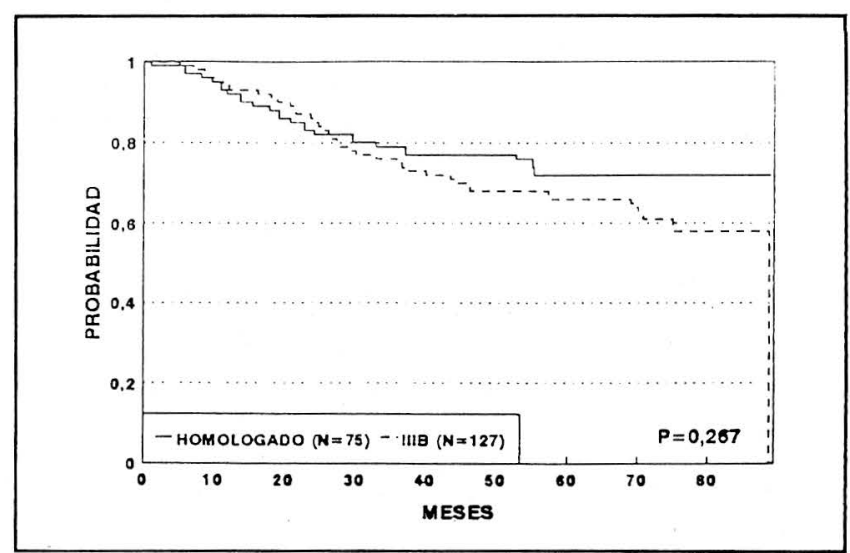


CARCINOMA DE SENO IIIB. TIEMPO LIBRE DE ENFERMEDAD SEGUN CLASIFICACION CLINICA

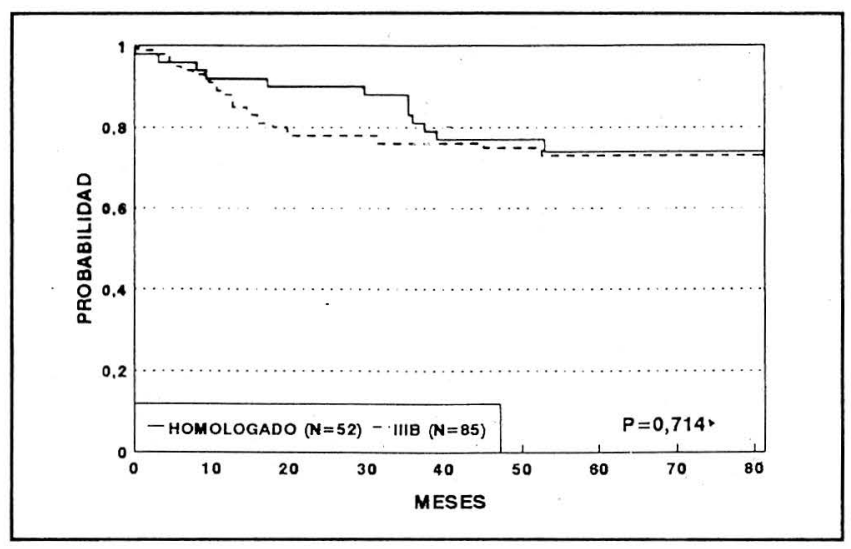

\section{Discusión}

El cáncer de seno es la segunda causa de consulta del Instituto Nacional de Cancerología, de estos pacientes la mayoría se encuentran en el estado IIIB (12-13), sinembargo hay un grupo importante de pacientes que antes de llegar a la consulta han sido manipulados sobre el tumor con objetivos diagnósticos o terapéuticos. En el INC, basados en experiencia histórica que los pacientes manipulados presentan menor período libre de enfermedad y sobrevida al compararlos con los estados correspondientes, se decidió clasificar a estos pacientes como Tx y se homologan al estado localmente más avanzado o IIIB. Revisando el Medline desde enero de 1985 hasta mayo de 1996 y en el Oncodisc desde enero de 1994 hasta mayo de 1996 no se encontraron artículos que describan la variable Tx como sinónimo tumor manipulado. En la literatura $T x$ eș una clasificación patológica que describe los tumores con el margen de sección quirúrgica macroscópicamente positivo. La clasificación Tx y su correspondiente homologación a IIIB es propia del Instituto Nacional de Cancerología de Colombia. Existe la preocupación por la posibilidad que estos pacientes homologados se encuentren en un estado clínico diferente al estado IIIB.

El presente es un estudio descriptivo retrospectivo diseñado con el fin de encontrar diferencias en relación con sobrevida, período libre de enfermedad y metástasis entre los pacientes que al momento del ingreso de la consulta de seno, entre las fechas del estudio, se encontraron en un estado clínico IIIB y los homologados por manipulación previa. Se encontró que los pacientes manipulados que fueron homologados al estado IIIB eran más jóvenes que los pacientes del correspondiente estado clínico. Sin embargo, no se encontró ninguna diferencia en cuanto al tamaño del tumor al momento del ingreso, características clínicas de $\mathrm{T} 4$ y compromiso axilar. Ambos grupos presentaron características histológicas y patológicas similares. No se encontró diferencia estadística cuando se compararon ambos grupos en relación a porcentaje de metástasis, período libre de enfermedad y sobrevida. Al analizar los hallazgos histopatológicos con período libre de enfermedad y recurrencia no se encontró diferencia entre los dos grupos descritos.

Preocupa que no fue posible reclasificar a los pacientes al momento de la manipulación para saber si hay un deterioro en su estado clínico durante el tiempo de remisión, porque la gran mayoría de los pacientes no tuvieron una clasificación clínica previa al momento de la manipulación y la hoja de remisión no tenía los datos adecuados para una clasificación TNM. Al intentar reclasificar los pacientes al momento del ingreso según el TNM se encontró que en el $40 \%$ de los casos no fue posible por falta de alguna de las características clínicas necesarias para el TNM y el otro $40 \%$ presentaban características clínicas de $\mathrm{T} 4$ y fueron reclasificados como IIIB. En el $80 \%$ de los pacientes de este último subgrupo no fue posible distinguir si el edema de piel se debía a la manipulación quirúrgica previa o a la infiltración tumoral; sinembargo, al compararlos como subgrupo con los IIIB clínicos y los demás Tx-IIIB se comportaron iguales en cuanto a sobrevida y período libre de enfermedad. Un $20 \%$ de los pacientes homologados tenían características TNM al momento del ingreso con las que se les podía clasificar en un estado más temprano; encontrándose diferencia en cuanto a sobrevida al compararlas con los IIIB, y menor índice de recaída como subgrupo.

Al momento de la manipulación el médico no tuvo en cuenta la clasificación TNM en 66 pacientes, en los 10 pacientes que al momento de la manipulación el médico identificó los factores TNM sólo tres permanecieron con el mismo estado al momento del ingreso (dos IIIB y un estado uno), en dos no se les pudo clasificar según el TNM por no encontrarse masa ni características de T4 y en los cinco restantes cambiaron el estado para uno más avanzado al momento del ingreso.

Por último no se encontró diferencia en relación con la sobrevida al comparar los estados IIIB con los Tx-IIIB teniendo en cuenta el tiempo transcurrido entre la manipulación y el momento del ingreso, sinembárgo esto no indica que la manipulación afecte o no la sobrevida de estos pacientes ya que desconocemos en el $89 \%$ de los casos el estado clínico del paciente al momento de la manipulación y el número de los pacientes restantes no soportan un análisis estadístico confiable.

A pesar de no poderse identificar el estado clínico al momento de la manipulación en un número importante de pacientes y al analizar los datos al momento del ingreso en la más de la mitad de éstos no se encontraron signos de T4 y que en el $80 \%$ de los restantes se identificaron como $\mathrm{T} 4$ por edema de piel indistinguible entre manipulación quirúrgica e infiltración tumoral, en este estudio se encontró que los pacientes homologados a IIIB por manipulación previa como grupo tienen un comportamiento similar al estado IIIB.

\section{Conclusión}

En el presente estudio no se puede concluir que haya diferencia en cuanto a sobrevida y período libre de enfermedad entre los pacientes con Ca de Seno estado IIIB y los pacientes homologados a este estado por haber sido manipulados fuera de la institución previa a su remisión para tratamiento. 


\section{BIBLIOGRAFIA}

1. Jay R., Harris JR., Veronessi U. Breast Cáncer. New England J.M. 1992; 327: 319-326.

2. Fox MS. JAMA $1979 ; 241: 489-494$.

3. Fisher B., Redmon C., Fisher ER et al. The Contribution to the NSABP clinical trials of primary breast Ca Therapy to an understanding of tumor Biology. Cancer 1980; 46: 1909-1025.

4. Fisher ER. NCI Monography 1986; 1: 29-34.

5. Yeatman TJ., Bland KI. Clinical and pathological staging of Breast CA. In The Breast, Coopeland E, Bland KI, Editors. Saunders, 1991; 313-330.

6. Fisher ER., Redmon C. Pathologic findings of invasive Breast $\mathrm{Ca}$, determinants for 5 year treatment failure (From the NSABP Protocol 4). Cancer 1980; 46: 908-918.
7. Swain SM., Lippman ME. Locally advanced Breast Cancer, in The Breast Coopeland E, Bland KI, Editors. Saunders, 1991; 843-862.

8. Osborne MP., Borgen P. Utilidad de la Mastectomía en el Cáncer mamario. Clin. Quir. NA 1990; 5: 1029-1054.

9. Cady B., Stone M. Selección de métodos para conservación mamaria en el carcinoma invasor primario. Clin. Quir. NA 1990; 5: 1055-1068.

10. Swain SM. Selección del tratamiento para el cáncer mamario en estado III. Clin. Quir. NA 1990; 5: 1069-1088.

11. Cáncer de Seno. En protocolos de manejo del cáncer. INC. Llamas Agusto, Editor INC 1992.

12. Informe de registro. Casos nuevos de cáncer en 1994 en INC. División de registro y seguimiento INC. Biblioteca INC.

13. Informe de registro. Casos nuevos de cáncer en 1995 en INC. División de registro y seguimiento INC. Biblioteca INC.

\section{PUBLICACIONES}

Informamos a nuestros lectores que en la oficina de la Revista Colombiana de Obstetricia y Ginecología se encuentran a disposición para consulta las siguientes revistas:

- International Journal of Gynecology \& Obstetrics

- Journal Brasilero de Ginecología

- Anales Chirurgiae et Gynaecologiae

- World Health Organization - Challenges in Reproductive Helth research

- Women's groups. NGOS and Safe Motherhood

- Revista Ecuatoriana de Ginecología y Obstetricia

- International Family Planning Perspectives

- Toko Ginecología Práctica

- Manual de Administración en Planificación Familiar

- Revista Obstetricia y Ginecología de Venezuela

- Revista Centroamericana de Obstetricia y Ginecología 